\title{
Article
}

\section{Fixed Points Results for Various Types of Tricyclic Contractions}

\author{
Mustapha Sabiri, Abdelhafid Bassou *, Jamal Mouline and Taoufik Sabar
}

check for

updates

Citation: Sabiri, M.; Bassou, A.; Mouline, J.; Sabar, T. Fixed Points Results for Various Types of Tricyclic Contractions. Axioms 2021, 10, 72. https://doi.org/10.3390/ axioms10020072

Academic Editor: Erdal Karapinar

Received: 8 March 2021

Accepted: 8 April 2021

Published: 20 April 2021

Publisher's Note: MDPI stays neutral with regard to jurisdictional claims in published maps and institutional affiliations.

Copyright: (c) 2021 by the authors. Licensee MDPI, Basel, Switzerland. This article is an open access article distributed under the terms and conditions of the Creative Commons Attribution (CC BY) license (https:// creativecommons.org/licenses/by/ $4.0 /)$.
Laboratory of Algebra, Analysis and Applications (L3A), Departement of Mathematics and Computer Science, Faculty of Sciences Ben M'sik, Hassan II University of Casablanca, Casablanca 20000, Morocco; sabiri10mustapha@gmail.com (M.S.); mouline61@gmail.com (J.M.); sabarsaw@gmail.com (T.S.)

* Correspondence: hbassou@gmail.com

Abstract: In this paper, we introduce four new types of contractions called in this order Kanan-Stype tricyclic contraction, Chattergea-S-type tricyclic contraction, Riech-S-type tricyclic contraction, Cirić-S-type tricyclic contraction, and we prove the existence and uniqueness for a fixed point for each situation.

Keywords: fixed points; S-type tricyclic contraction; metric spaces

\section{Introduction}

It is well known that the Banach contraction principle was published in 1922 by S. Banach as follows:

Theorem 1. Let $(X, d)$ be a complete metric space and a self mapping $T: X \longrightarrow X$. If there exists $k \in[0,1)$ such that, for all $x, y \in X, d(T x, T y) \leq k d(x, y)$, then $T$ has a unique fixed point in $X$.

The Banach contraction principle has been extensively studied and different generalizations were obtained.

In 1968 [1], Kannan established his famous extension of this contraction.

Theorem 2. Ref. [1] Let $(X, d)$ be a complete metric space and a self mapping $T: X \longrightarrow X$. If $T$ satisfies the following condition:

$$
d(T x, T y) \leq k[d(x, T x)+d(y, T y)] \quad \text { for all } \quad x, y \in X \quad \text { where } 0<k<\frac{1}{2}
$$

then $T$ has a fixed point in $X$.

A similar contractive condition has been introduced by Chattergea in 1972 [2] as follows:

Theorem 3. Ref. [2] Let $T: X \longrightarrow X$, where $(X, d)$ is a complete metric space. If there exists $0<k<\frac{1}{2}$ such that

$$
d(T x, T y) \leq k[d(y, T x)+d(T y, x)] \text { for all } x, y \in X
$$

then $T$ has a fixed point in $X$.

We can also find another extension of the Banach contraction principle obtained by S. Reich, Kannan in 1971 [3].

Theorem 4. Ref. [3] Let $T: X \longrightarrow X$, where $(X, d)$ is a complete metric space. If there exists $0<k<\frac{1}{3}$ such that

$$
d(T x, T y) \leq k[d(x, y)+d(x, T x)+d(y, T y)] \text { for all } \quad x, y \in X
$$


then $T$ has a fixed point in $X$.

In addition, in the same year, Cirić gave the following extension [4].

Theorem 5. Ref. [4] Let $T: X \longrightarrow X$, where $(X, d)$ a complete metric space. If there exists $k \in[0,1)$ such that

$$
d(T x, T y) \leq k M a x[d(x, y), d(x, T x), d(y, T y), d(y, T x), d(T y, x)] \text { for all } x, y \in X,
$$

then $T$ has a fixed point in $X$.

Many authors have investigated these situations and many results were proved (see [5-13]).

In this article, we prove the uniqueness and existence of the fixed points in different types contractions for a self mapping $T$ defined on the union of tree closed subsets of a complete metric space with $k$ in different intervals.

\section{Preliminaries}

In best approximation theory, the concept of tricyclic mappings extends that of ordinary cyclic mappings. Moreover, in the case where two of the sets, say A and C, coincide, we find a cyclic mapping which is also a self-map, and, hence, a best proximity point result for a tricyclic mappings means also a fixed point and a best proximity point result for a self-map and a cyclic mapping.

Definition 1. Let $A, B$ be nonempty subsets of a metric space $(X, d)$. A mapping $T: A \cup B \longrightarrow$ $A \cup B$ is said to be cyclic if :

$$
T(A) \subseteq B, T(B) \subseteq A .
$$

In 2003, Kirk et al. [14] proved that, if $T: A \cup B \longrightarrow A \cup B$ is cyclic and, for some $k \in(0,1), d(T x, T y) \leq k d(x, y)$ for all $x \in A, y \in B$, then $A \cap B \neq \varnothing$, and $T$ has a unique fixed point in $A \cap B$.

In 2017, Sabar et al. [15] proved a similar result for tricyclic mappings and introduced the concept of tricyclic contractions.

Theorem 6. Ref. [15] Let $A, B$ and $C$ be nonempty closed subsets of a complete metric space $(X, d)$, and let a mapping $T: A \cup B \cup C \longrightarrow A \cup B \cup C$. If $T(A) \subseteq B, T(B) \subseteq C$ and $T(C) \subseteq A$ and there exists $k \in(0,1)$ such that $D(T x, T y, T z) \leq k D(x, y, z)$ for all $(x, y, z) \in A \times B \times C$, then $A \cap B \cap C$ is nonempty and $T$ has a unique fixed point in $A \cap B \cap C$,

where $D(x, y, z)=d(x, y)+d(x, z)+d(y, z)$.

Definition 2. Ref. [15] Let $A, B$ and $C$ be nonempty subsets of a metric space $(X, d)$. A mapping $T: A \cup B \cup C \longrightarrow A \cup B \cup C$ is said to be tricyclic contracton if there exists $0<k<1$ such that:

1. $T(A) \subseteq B, T(B) \subseteq C$ and $T(C) \subseteq A$.

2. $D(T x, T y, T z) \leq k D(x, y, z)+(1-k) \delta(A, B, C)$ for all $(x, y, z) \in A \times B \times C$.

where $\delta(A, B, C)=\inf \{D(x, y, z): x \in A, y \in B, z \in C\}$

Very Recently, Sabiri et al. introduced an extension of the aforementioned mappings and called them p-cyclic contractions [16].

\section{Main Results}

Definition 3. Let $A, B$ and $C$ be nonempty subsets of a metric space $(X, d)$. A mapping $T$ : $A \cup B \cup C \longrightarrow A \cup B \cup C$ is said to be a Kannan-S-type tricyclic contraction, if there exists $k \in\left(0, \frac{1}{3}\right)$ such that 
1. $T(A) \subseteq B, T(B) \subseteq C, T(C) \subseteq A$.

2. $D(T x, T y, T z) \leq \bar{k}[d(x, T x)+d(y, T y)+d(z, T z)]$ for all $(x, y, z) \in A \times B \times C$.

We give an example to show that a map can be a tricyclic contraction but not a Kannan-S-type tricyclic contraction.

Example 1. Let $X$ be $\mathbb{R}^{2}$ normed by the norm $\|(x, y)\|=|x|+|y|$, and $A=[1,2] \times\{0\}, B=$ $\{0\} \times[-2,-1], C=[-2,-1] \times\{0\}$, then

$$
\delta(A, B, C)=D((1,0),(0,-1),(-1,0))=6 .
$$

Put $T: A \cup B \cup C \longrightarrow A \cup B \cup C$ such that

$$
\begin{array}{ll}
T(x, 0)=\left(0,-\frac{x+2}{3}\right) & \text { if }(x, 0) \in A, \\
T(0, y)=\left(\frac{y-2}{3}, 0\right) & \text { if }(0, y) \in B, \\
T(z, 0)=\left(-\frac{z-2}{3}, 0\right) & \text { if }(z, 0) \in C,
\end{array}
$$

We have $T(A) \subseteq B, T(B) \subseteq C$ and $T(C) \subseteq A$, and

$$
\begin{aligned}
D(T(x, 0), T(0, y), T(z, 0)) & =D\left(\left(0,-\frac{x+2}{3}\right),\left(\frac{y-2}{3}, 0\right),\left(-\frac{z-2}{3}, 0\right)\right) \\
& =\frac{2}{3}(x-y-z)+4 \\
& =\frac{1}{3} D((x, 0),(0, y),(z, 0))+4 \\
& =\frac{1}{3} D((x, 0),(0, y),(z, 0))+\left(1-\frac{1}{3}\right) \delta(A, B, C)
\end{aligned}
$$

for all $(x, 0) \in A,(0, y) \in B,(z, 0) \in C$.

On the other hand,

$$
D(T(2,0), T(0,-2), T(-2,0))=D\left(\left(0,-\frac{4}{3}\right),\left(\frac{-4}{3}, 0\right),\left(\frac{4}{3}, 0\right)\right)=8
$$

and

$$
d((2,0), T(2,0))+d((0,-2), T(0,-2))+d((-2,0), T(-2,0))=10
$$

which implies that

$D(T(2,0), T(0,-2), T(-2,0))$

$$
>\frac{1}{3}[d((2,0), T(2,0))+d((0,-2), T(0,-2))+d((-2,0), T(-2,0))]
$$

Then, $T$ is tricyclic contraction but not a Kannan-S-type tricyclic contraction.

Now, we give an example for which $T$ is a Kannan-S-type tricyclic contraction but not a tricyclic contraction.

Example 2. Let $X=\mathbb{R}$ with the usual metric. Let $A=B=C=[0,1]$, then $\delta(A, B, C)=0$. Put $T: A \cup B \cup C \longrightarrow A \cup B \cup C$ such that

$$
\text { Tx }=\frac{1}{6} \text { if } 0 \leq x<1, \quad \text { Tx }=\frac{1}{4} \text { if } x=1
$$


For $x=1, y=1$ and $z=\frac{23}{24}$, we have

$$
D\left(T(1), T(1), T\left(\frac{23}{24}\right)\right)=D\left(\frac{1}{4}, \frac{1}{4}, \frac{1}{6}\right)=2 d\left(\frac{1}{4}, \frac{1}{6}\right)=\frac{1}{6} .
$$

and

$$
D\left(1,1, \frac{23}{24}\right)=2 d\left(1, \frac{23}{24}\right)=\frac{1}{12} .
$$

Then, $T$ is not tricyclic contraction.

However $T$ is a Kannan-S-type tricyclic contraction. Indeed:

- If $x=y=z=1$, we have

$$
D(T(1), T(1), T(1))=0 \leq \frac{9}{4} k
$$

for all $k \geq 0$, then for $0 \leq k<\frac{1}{3}$.

- If $x \in[0,1), y \in[0,1)$ and $z \in[0,1)$, we have

$$
D(T x, T y, T z)=0 \leq k\left(d\left(x, \frac{1}{6}\right)+d\left(y, \frac{1}{6}\right)+d\left(z, \frac{1}{6}\right)\right.
$$

for all $k \geq 0$, then for $0 \leq k<\frac{1}{3}$.

- If $x=1, y \in[0,1)$ and $z \in[0,1)$, we have

$$
D\left(T_{1}, T y, T z\right)=D\left(\frac{1}{4}, \frac{1}{6}, \frac{1}{6}\right)=\frac{1}{6}
$$

and

$$
d(1, T(1))+d(y, T y)+d(z, T z)=\frac{3}{4}+d\left(y, \frac{1}{6}\right)+d\left(z, \frac{1}{6}\right),
$$

then, for $k=\frac{2}{9}$, we have

$$
D(T(1), T(y, T z) \leq k(d(1, T(1))+d(y, T y)+d(z, T z)) .
$$

- If $x=1, y=1$ and $z \in[0,1)$, we have

$$
D(T(1), T(1), T z)=D\left(\frac{1}{4}, \frac{1}{4}, \frac{1}{6}\right)=\frac{1}{6}
$$

and

$$
d(1, T(1))+d(1, T(1))+d(z, T z)=\frac{3}{2}+d\left(z, \frac{1}{6}\right) .
$$

Then, for $k=\frac{2}{9}$, we have

$$
D(T(1), T(1), T z) \leq k(d(1, T(1))+d(1, T(1))+d(z, T z)) .
$$

Consequently, for $k=\frac{2}{9}$, we have:

$$
D(T x, T y, T z) \leq k(d(x, T x)+d(y, T y)+d(z, T z)) \text { for all }(x, y, z) \in A \times B \times C .
$$

Theorem 7. Let $A, B$ and $C$ be nonempty closed subsets of a complete metric space $(X, d)$, and let $T: A \cup B \cup C \longrightarrow A \cup B \cup C$ be a Kannan-S-type tricyclic contraction. Then, $T$ has a unique fixed point in $A \cap B \cap C$.

Proof. Fix $x \in A$. We have

$$
d\left(T^{3} x, T^{2} x\right) \leq D\left(T^{3} x, T^{2} x, T x\right) \leq k\left[d\left(T^{2} x, T^{3} x\right)+d\left(T x, T^{2} x\right)+d(x, T x)\right] .
$$


Then,

$$
d\left(T^{3} x, T^{2} x\right) \leq k\left[d\left(T^{2} x, T^{3} x\right)+d\left(T x, T^{2} x\right)+d(x, T x)\right],
$$

which implies

$$
d\left(T^{3} x, T^{2} x\right) \leq \frac{k}{(1-k)}\left[d\left(T x, T^{2} x\right)+d(x, T x)\right] .
$$

Similarly, we have

$$
\begin{gathered}
d\left(T^{2} x, T x\right) \leq \frac{k}{(1-k)}\left[d\left(T^{3} x, T^{2} x\right)+d(x, T x)\right] \\
d\left(T^{2} x, T x\right) \leq \frac{k}{(1-k)}\left[\frac{k}{(1-k)}\left[d\left(T x, T^{2} x\right)+d(x, T x)\right]+d(x, T x)\right] \\
\Longrightarrow d\left(T^{2} x, T x\right) \leq \frac{k}{1-2 k}(d(x, T x))
\end{gathered}
$$

Then,

$$
d\left(T^{2} x, T x\right) \leq t d(x, T x) \text { where } t=\frac{k}{1-2 k} \text { and } t \in(0,1),
$$

which implies

$$
d\left(T^{n+1} x, T^{n} x\right) \leq t^{n} d(x, T x) \text {, for all } n \geq 1
$$

Consequently,

$$
\sum_{n=1}^{+\infty} d\left(T^{n+1} x, T^{n} x\right) \leq\left(\sum_{n=1}^{+\infty} t^{n}\right) d(x, T x)<+\infty
$$

implies that $\left\{T^{n} x\right\}$ is a Cauchy sequence in $(X, d)$. Hence, there exists $z \in A \cup B \cup C$ such that $T^{n} x \longrightarrow z$. Notice that $\left\{T^{3 n} x\right\}$ is a sequence in $A,\left\{T^{3 n-1} x\right\}$ is a sequence in $C$ and $\left\{T^{3 n-2} x\right\}$ is a sequence in $B$ and that both sequences tend to the same limit $z$. Regarding the fact that $A, B$ and $C$ are closed, we conclude $z \in A \cap B \cap C$, hence $A \cap B \cap C \neq \varnothing$. To show that $z$ is a fixed point, we must show that $T z=z$. Observe that

$$
\begin{aligned}
d(T z, z) & =\lim d\left(T z, T^{3 n} x\right) \leq \lim D\left(T^{3 n} x, T^{3 n-1} x, T z\right) \\
& \leq \lim k\left[d\left(T^{3 n-1} x, T^{3 n} x\right)+d\left(T^{3 n-2} x, T^{3 n-1} x\right)+d(z, T z)\right] \\
& \leq k d(T z, z)
\end{aligned}
$$

which is equivalent to

$$
(1-k) d(T z, z)=0
$$

Since $k \in\left(0, \frac{1}{3}\right)$, then $d(T z, z)=0$, which implies $T z=z$.

To prove the uniqueness of $z$, assume that there exists $w \in A \cup B \cup C$ such that $w \neq z$ and $T w=w$. Taking into account that $T$ is tricyclic, we get $w \in A \cap B \cap C$. We have

$$
d(z, w)=d(T z, T w) \leq D(T z, T w, T w) \leq k[d(z, T z)+d(w, T w)+d(w, T w)]=0
$$

which implies $d(z, w)=0$. We get that $z=w$ and hence $z$ is the unique fixed point of $T$.

Example 3. Let $X$ be $\mathbb{R}^{2}$ normed by the norm $\|(x, y)\|=|x|+|y|$, let $A=\{0\} \times[0,+1], B=$ $[0,+1] \times\{0\}, C=\{0\} \times[-1,0]$ and let $T: A \cup B \cup C \longrightarrow A \cup B \cup C$ be defined by

$$
\begin{array}{ll}
T(0, x)=\left(\frac{x}{6}, 0\right) & \text { if }(0, x) \in A, \\
T(y, 0)=\left(0, \frac{-y}{6}\right) & \text { if }(y, 0) \in B,
\end{array}
$$




$$
T(0, z)=\left(0, \frac{-z}{6}\right) \quad \text { if }(0, z) \in C
$$

We have

$$
T(A) \subseteq B, T(B) \subseteq C \text { and } T(C) \subseteq A
$$

In addition, for all $(0, x) \in A,(y, 0) \in B,(0, z) \in C$, we have

$$
D(T(0, x), T(y, 0), T(0, z))=D\left(\left(\frac{x}{6}, 0\right),\left(0, \frac{-y}{6}\right),\left(0, \frac{-z}{6}\right)\right)=\frac{1}{3}(x+y-z)
$$

In addition, we have

$$
d((0, x), T(0, x))+d((y, 0), T(y, 0))+d((0, z), T(0, z))=\frac{7}{6}(x+y-z)
$$

This implies

$$
D(T(0, x), T(y, 0), T(0, z))=\frac{2}{7}[d((0, x), T(0, x))+d((y, 0), T(y, 0))+d((0, z), T(0, z))] .
$$

Then, $T$ is a Kannan-S-type tricyclic contraction, and $T$ has a unique fixed point $(0,0)$ in $A \cap B \cap C$.

Corollary 1. Let $(X, d)$ be a complete metric space and a self mapping $T: X \longrightarrow X$. If there exists $k \in\left(0, \frac{1}{3}\right)$ such that

$$
D(T x, T y, T z) \leq k[d(x, T x)+d(y, T y)+d(z, T z)]
$$

for all $(x, y, z) \in X^{3}$, then $T$ has a unique fixed point.

Now, we shall define another type of a tricyclic contraction.

Definition 4. Let $A, B$ and $C$ be nonempty subsets of a metric space $(X, d)$. A mapping $T: A \cup$ $B \cup C \longrightarrow A \cup B \cup C$ is said to be a Chattergea-S-type tricyclic contraction if $T(A) \subseteq B, T(B) \subseteq$ $C, T(C) \subseteq A$, and there exist $k \in\left(0, \frac{1}{3}\right)$ such that $D(T x, T y, T z) \leq k[d(y, T x)+d(z, T y)+d(x, T z)]$ for all $(x, y, z) \in A \times B \times C$.

Theorem 8. Let $A, B$ and $C$ be nonempty closed subsets of a complete metric space $(X, d)$, and let $T: A \cup B \cup C \longrightarrow A \cup B \cup C$ be a Chattergea-S-type tricyclic contraction. Then, T has a unique fixed point in $A \cap B \cap C$.

Proof. Fix $x \in A$. We have

$$
D\left(T x, T^{2} x, T^{3} x\right) \leq k\left[d(T x, T x)+d\left(T^{2} x, T^{2} x\right)+d\left(T^{3} x, x\right)\right]
$$

which implies

$$
D\left(T^{3} x, T^{2} x, T x\right) \leq k d\left(T^{3} x, x\right)
$$

so

$$
\begin{gathered}
d\left(T^{3} x, T^{2} x\right) \leq k\left[d\left(T^{3} x, T^{2} x\right)+d\left(T^{2} x, T x\right)+d(T x, x)\right] \text { (by the triangular inequality) } \\
\Longrightarrow d\left(T^{3} x, T^{2} x\right) \leq \frac{k}{(1-k)}\left[d\left(T x, T^{2} x\right)+d(x, T x)\right]
\end{gathered}
$$

and

$$
d\left(T^{2} x, T x\right) \leq D\left(T^{3} x, T^{2} x, T x\right) \leq \frac{k}{(1-k)}\left[d\left(T^{3} x, T^{2} x\right)+d(x, T x)\right]
$$




$$
\begin{aligned}
\Longrightarrow d\left(T^{2} x, T x\right) \leq & \frac{k}{(1-k)}\left[\frac{k}{(1-k)}\left[d\left(T x, T^{2} x\right)+d(x, T x)\right]+d(x, T x)\right] \\
& \Longrightarrow d\left(T^{2} x, T x\right) \leq \frac{k}{1-2 k}(d(x, T x))
\end{aligned}
$$

Then,

$$
d\left(T^{2} x, T x\right) \leq t d(x, T x) \text { where } t=\frac{k}{1-2 k} \text { and } t \in(0,1),
$$

which implies

$$
d\left(T^{n+1} x, T^{n} x\right) \leq t^{n} d(x, T x)
$$

for all $n \geq 1$. Consequently,

$$
\sum_{n=1}^{+\infty} d\left(T^{n+1} x, T^{n} x\right) \leq\left(\sum_{n=1}^{+\infty} t^{n}\right) d(x, T x)<+\infty
$$

implies that $\left\{T^{n} x\right\}$ is a Cauchy sequence in $(X, d)$. Hence, there exists $z \in A \cup B \cup C$ such that $T^{n} x \longrightarrow z$. Notice that $\left\{T^{3 n} x\right\}$ is a sequence in $A,\left\{T^{3 n-1} x\right\}$ is a sequence in $C$, and $\left\{T^{3 n-2} x\right\}$ is a sequence in $B$ and that both sequences tend to the same limit $z$. Regarding that $A, B$ and $C$ are closed, we conclude $z \in A \cap B \cap C$, hence $A \cap B \cap C \neq \varnothing$.

To show that $z$ is a fixed point, we must show that $T z=z$. Observe that

$$
\begin{aligned}
d(T z, z) & =\lim d\left(T z, T^{3 n} x\right) \leq \lim D\left(T z, T^{3 n} x, T^{3 n-1} x\right) \\
& \leq \lim k\left[d\left(T^{3 n-1} x, T z\right)+\left(T^{3 n-2} x, T^{3 n} x\right)+d\left(z, T^{3 n-1} x\right)\right] \leq k d(T z, z),
\end{aligned}
$$

which is equivalent to $(1-k) d(T z, z)=0$. Since $k \in\left(1, \frac{1}{3}\right)$, then $d(T z, z)=0$, which implies $T z=z$.

To prove the uniqueness of $z$, assume that there exists $w \in A \cup B \cup C$ such that $w \neq z$ and $T w=w$. Taking into account that $T$ is tricyclic, we get $w \in A \cap B \cap C$.

We have

$$
\begin{aligned}
d(z, w) & =d(T z, T w) \leq D(T z, T w, T w) \\
& \leq k[d(T z, w)+d(T w, w)+d(T w, z)] \\
& \leq 2 k d(z, w) .
\end{aligned}
$$

Then, $d(z, w)=0$. We conclude that $z=w$ and hence $z$ is the unique fixed point of $T$.

Corollary 2. Let $(X, d)$ be a complete metric space and a self mapping $T: X \longrightarrow X$. If there exists $k \in\left(0, \frac{1}{3}\right)$ such that

$$
D(T x, T y, T z) \leq k[d(y, T x)+d(z, T y)+d(x, T z)]
$$

for all $(x, y, z) \in X^{3}$, then $T$ has a unique fixed point.

In this step, we define a Reich-S-type tricyclic contraction.

Definition 5. Let $A, B$ and $C$ be nonempty subsets of a metric space $(X, d)$.

A mapping $T: A \cup B \cup C \longrightarrow A \cup B \cup C$ is said to be a Reich-S-type tricyclic contraction if there exists $k \in\left(0, \frac{1}{7}\right)$ such that:

1. $T(A) \subseteq B, T(B) \subseteq C, T(C) \subseteq A$. 
2. $D(T x, T y, T z) \leq k[D(x, y, z)+d(x, T x)+d(y, T y)+d(z, T z)]$ for all $(x, y, z) \in A \times$ $B \times C$.

Theorem 9. Let $A, B$ and $C$ be nonempty closed subsets of a complete metric space $(X, d)$, and let $T: A \cup B \cup C \longrightarrow A \cup B \cup C$ be a Reich-S-type tricyclic contraction. Then, Thas a unique fixed point in $A \cap B \cap C$.

Proof. Fix $x \in A$. We have

$$
\begin{aligned}
& d\left(T^{2} x, T^{3} x\right) \leq D\left(T x, T^{2} x, T^{3} x\right) \\
& \leq k\left[D\left(x, T x, T^{2} x\right)+d\left(T^{2} x, T^{3} x\right)+d\left(T x, T^{2} x\right)+d(x, T x)\right] \\
& \Longrightarrow d\left(T^{2} x, T^{3} x\right)(1-k) \leq k\left[2 d\left(T^{2} x, T x\right)+2 d(x, T x)+d\left(T^{2} x, x\right)\right] \\
& \Longrightarrow d\left(T^{2} x, T^{3} x\right) \leq \\
& \leq \frac{k}{1-k}\left[2 d\left(T^{2} x, T x\right)+2 d(x, T x)+d\left(T^{2} x, x\right)\right] \\
& \leq \frac{k}{1-k}\left[3 d\left(T^{2} x, T x\right)+3 d(x, T x)\right] \\
& \Longrightarrow d\left(T^{2} x, T^{3} x\right) \leq \frac{3 k}{1-k}\left[d\left(T^{2} x, T x\right)+d(x, T x)\right]
\end{aligned}
$$

and

$$
\begin{gathered}
d\left(T^{2} x, T x\right) \leq D\left(T x, T^{2} x, T^{3} x\right) \leq k\left[D\left(x, T x, T^{2} x\right)+d\left(T^{2} x, T^{3} x\right)+d\left(T x, T^{2} x\right)+d(x, T x)\right] \\
\Longrightarrow d\left(T^{2} x, T x\right) \leq k\left[3 d\left(T^{2} x, T x\right)+3 d(x, T x)+d\left(T^{2} x, T^{3} x\right)\right] \\
\Longrightarrow d\left(T^{2} x, T x\right)(1-3 k) \leq k\left[d\left(T^{2} x, T^{3} x\right)+3 d(x, T x)\right] \\
\Longrightarrow d\left(T^{2} x, T x\right) \leq \frac{k}{1-3 k} d\left(T^{2} x, T^{3} x\right)+\frac{3 k}{1-3 k} d(x, T x) \\
\Longrightarrow d\left(T^{2} x, T x\right) \leq \frac{k}{1-3 k} \frac{3 k}{1-k}\left[d\left(T^{2} x, T x\right)+d(x, T x)\right]+\frac{3 k}{1-3 k} d(x, T x) \\
\Longrightarrow d\left(T^{2} x, T x\right) \leq \frac{3 k^{2}}{(1-3 k)(1-k)} d\left(T^{2} x, T x\right)+\left(\frac{3 k^{2}}{(1-3 k)(1-k)}+\frac{3 k}{(1-3 k)}\right) d(x, T x) \\
\Longrightarrow d\left(T^{2} x, T x\right)\left(1-\frac{3 k^{2}}{(1-3 k)(1-k)}\right) \leq \frac{3 k^{2}+3 k(1-k)}{(1-3 k)(1-k)} d(x, T x) \\
\Longrightarrow d\left(T^{2} x, T x\right)\left((1-3 k)(1-k)-3 k^{2}\right) \leq\left(3 k^{2}+3 k(1-k)\right) d(x, T x) \\
\Longrightarrow d\left(T^{2} x, T x\right)(1-4 k) \leq 3 k d(x, T x) \\
\Longrightarrow d\left(T^{2} x, T x\right) \leq \frac{3 k}{(1-4 k)} d(x, T x) .
\end{gathered}
$$

Then,

$$
d\left(T^{2} x, T x\right) \leq t d(x, T x) \text { where } t=\frac{3 k}{(1-4 k)} \text { and } t \in(0,1),
$$

which implies

$$
d\left(T^{n+1} x, T^{n} x\right) \leq t^{n} d(x, T x)
$$


consequently

$$
\sum_{n=1}^{+\infty} d\left(T^{n+1} x, T^{n} x\right) \leq\left(\sum_{n=1}^{+\infty} t^{n}\right) d(x, T x)<+\infty
$$

This implies that $\left\{T^{n} x\right\}$ is a Cauchy sequence in $(X, d)$. Hence, there exists $z \in$ $A \cup B \cup C$ such that $T^{n} x \longrightarrow z$. Notice that $\left\{T^{3 n} x\right\}$ is a sequence in $A,\left\{T^{3 n-1} x\right\}$ is a sequence in $C$ and $\left\{T^{3 n-2} x\right\}$ is a sequence in $B$ and that both sequences tend to the same limit $z$. Regarding the fact that $A, B$ and $C$ are closed, we conclude that $z \in A \cap B \cap C$, hence $A \cap B \cap C \neq \varnothing$.

To show that $z$ is a fixed point, we must show that $T z=z$. Observe that

$$
\begin{aligned}
d(T z, z) & =\lim d\left(T z, T^{3 n} x\right) \\
& \leq \lim D\left(T^{3 n} x, T^{3 n-1} x, T z\right) \\
& \leq \lim k\left[d\left(T^{3 n-1} x, T^{3 n-2} x\right)+d\left(T^{3 n-1} x, z\right)+d\left(T^{3 n-2} x, z\right)\right. \\
& \left.+d\left(T^{3 n-1} x, T^{3 n} x\right)+d\left(T^{3 n-2} x, T^{3 n-1} x\right)+d(z, T z)\right] \\
& \leq k d(T z, z),
\end{aligned}
$$

which is equivalent to $(1-k) d(T z, z)=0$.

Since $k \in\left(0, \frac{1}{7}\right)$, then $d(T z, z)=0$, which implies $T z=z$.

To prove the uniqueness of $z$, assume that there exists $w \in A \cup B \cup C$ such that $w \neq z$ and $T w=w$. Taking into account that $T$ is tricyclic, we get $w \in A \cap B \cap C$.

$$
\begin{aligned}
d(z, w) & =d(T z, T w) \\
& \leq D(T z, T w, T w) \\
& \leq k[2 d(z, w)+d(w, w)+d(z, T z)+d(T w, w)+d(T w, w)] \\
& \leq 2 k d(z, w)
\end{aligned}
$$

implies $d(z, w)=0$. We conclude that $z=w$ and hence $z$ is the unique fixed point of $T$.

Example 4. We take the same example 3.

Let $X$ be $\mathbb{R}^{2}$ normed by the norm $\|(x, y)\|=|x|+|y|$,

$$
A=\{0\} \times[0,+1], B=[0,+1] \times\{0\}, C=\{0\} \times[-1,0]
$$

and let $T: A \cup B \cup C \longrightarrow A \cup B \cup C$ be defined by

$$
\begin{aligned}
& T(0, x)=\left(\frac{x}{6}, 0\right) \quad \text { if }(0, x) \in A, \\
& T(y, 0)=\left(0, \frac{-y}{6}\right) \quad \text { if }(y, 0) \in B, \\
& T(0, z)=\left(0, \frac{-z}{6}\right) \quad \text { if }(0, z) \in C,
\end{aligned}
$$

We have $T$ is tricyclic and for all $(0, x) \in A,(y, 0) \in B,(0, z) \in C$,

$$
\begin{aligned}
D(T(0, x), T(y, 0), T(0, z)) & =D\left(\left(\frac{x}{6}, 0\right),\left(0, \frac{-y}{6}\right),\left(0, \frac{-z}{6}\right)\right) \\
& =\frac{1}{3}(x+y-z) .
\end{aligned}
$$


In addition, we have

$$
\begin{gathered}
D((0, x),(y, 0),(0, z))+d((0, x), T(0, x))+d((y, 0), T(y, 0))+d((0, z), T(0, z)) \\
=2(x+y-z)+\frac{7}{6}(x+y-z)=\frac{19}{6}(x+y-z) .
\end{gathered}
$$

Then,

$$
\begin{aligned}
D(T(0, x), T(y, 0), T(0, z))= & \frac{2}{19}(D((0, x),(y, 0),(0, z))+d((0, x), T(0, x)) \\
& +d((y, 0), T(y, 0))+d((0, z), T(0, z))) \\
\leq & \frac{1}{7}(D((0, x),(y, 0),(0, z))+d((0, x), T(0, x)) \\
& +d((y, 0), T(y, 0))+d((0, z), T(0, z)))
\end{aligned}
$$

This implies that $T$ is a Reich-S-type tricyclic contraction, and $T$ has a unique fixed point $(0,0)$ in $A \cap B \cap C$.

Corollary 3. Let $(X, d)$ a complete metric space and a self mapping $T: X \longrightarrow X$. If there exists $k \in\left(0, \frac{1}{7}\right)$ such that

$$
D(T x, T y, T z) \leq k[D(x, y, z)+d(x, T x)+d(y, T y)+d(z, T z)]
$$

for all $(x, y, z) \in X^{3}$, then $T$ has a unique fixed point in $X$.

The next tricyclic contraction considered in this section is the Cirić-S-type tricyclic contraction defined below.

Definition 6. Let $A, B$ and $C$ be nonempty subsets of a metric space $(X, d), T: A \cup B \cup C \longrightarrow$ $A \cup B \cup C$ be a Cirié-S-type tricyclic contraction, if there exists $k \in(0,1)$ such that

1. $T(A) \subseteq B, T(B) \subseteq C, T(C) \subseteq A$

2. $D(T x, T y, T z) \leq k M(x, y, z)$ for all $(x, y, z) \in A \times B \times C$.

where $M(x, y, z)=\max \{D(x, y, z), d(x, T x), d(y, T y), d(z, T z)\}$

The fixed point theorem of the Cirić-S-type tricyclic contraction reads as follows.

Theorem 10. Let $A, B$ and $C$ be nonempty closed subsets of a complete metric space $(X, d)$, and let $T: A \cup B \cup C \longrightarrow A \cup B \cup C$ be a Cirić-S-type tricyclic contraction, then $T$ has a unique fixed point in $A \cap B \cap C$.

Proof. Taking $x \in A$, we have $D(T x, T y, T z) \leq k M(x, y, z)$ for all $(x, y, z) \in A \times B \times C$. If $M(x, y, z)=D(x, y, z)$, Theorem 7 implies the desired result.

Consider the case $M(x, y, z)=d(x, T x)$. We have:

$$
\begin{aligned}
D\left(T x, T^{2} x, T^{3} x\right) \leq k d(x, T x) & \Longrightarrow d\left(T x, T^{2} x\right) \leq k d(x, T x) \\
& \Longrightarrow d\left(T^{n} x, T^{n+1} x\right) \leq k^{n} d(x, T x)
\end{aligned}
$$

Consequently,

$$
\sum_{n=1}^{+\infty} d\left(T^{n+1} x, T^{n} x\right) \leq\left(\sum_{n=1}^{+\infty} k^{n}\right) d(x, T x)<+\infty
$$

which implies that $\left\{T^{n} x\right\}$ is a Cauchy sequence in $(X, d)$. Hence, there exists $z \in A \cup B \cup C$ such that $T^{n} x \longrightarrow z$. Notice that $\left\{T^{3 n} x\right\}$ is a sequence in $A,\left\{T^{3 n-1} x\right\}$ is a sequence in $C$, 
and $\left\{T^{3 n-2} x\right\}$ is a sequence in $B$ and that both sequences tend to the same limit $z$; regarding the fact that $A, B$ and $C$ are closed, we conclude $z \in A \cap B \cap C$, hence $A \cap B \cap C \neq \varnothing$.

To show that $z$ is a fixed point, we must show that $T z=z$. Observe that

$$
d(T z, z)=\lim d\left(T z, T^{3 n} x\right) \leq \lim D\left(T^{3 n} x, T^{3 n-1} x, T z\right) \leq k d(T z, z),
$$

which is equivalent to $(1-k) d(T z, z)=0$. Since $k \in(0,1)$, then $d(T z, z)=0$, which implies $T z=z$.

To prove the uniqueness of $z$, assume that there exists $w \in A \cup B \cup C$ such that $w \neq z$ and $T w=w$.

Taking into account that $T$ is tricyclic, we get $w \in A \cap B \cap C$.

$d(z, w)=d(T z, T w) \leq D(T z, T w, T w) \leq k d(z, T z)=0$ implies $d(z, w)=0$. We conclude that $z=w$ and hence $z$ is the unique fixed point of $T$.

Consider the case $M(x, y, z)=d(y, T y)$. We have :

$$
D\left(T x, T^{2} x, T^{3} x\right) \leq k d\left(T x, T^{2} x\right) \Longrightarrow d\left(T x, T^{2} x\right) \leq k d\left(T x, T^{2} x\right)<d\left(T x, T^{2} x\right),
$$

which is impossible since $k \in(0,1)$

Consider the case $M(x, y, z)=d(z, T z)$. We have:

$$
D\left(T x, T^{2} x, T^{3} x\right) \leq k d\left(T^{2} x, T^{3} x\right) \Longrightarrow d\left(T^{2} x, T^{3} x\right) \leq k d\left(T^{2} x, T^{3} x\right)<d\left(T^{2} x, T^{3} x\right),
$$

which is impossible since $k \in(0,1)$.

Corollary 4. Let $A, B$ and $C$ be a nonempty subset of a complete metric space $(X, d)$ and let a mapping $T: A \cup B \cup C \longrightarrow A \cup B \cup C$. If there exists $k \in(0,1)$ such that

1. $T(A) \subseteq B, T(B) \subseteq C, T(C) \subseteq A$.

2. $D(T x, T y, T z) \leq k \max \{D(x, y, z), d(x, T x)\} \forall(x, y, z) \in A \times B \times C$.

Then, $T$ has a unique fixed point in $A \cap B \cap C$.

Author Contributions: Conceptualization, M.S. and A.B.; validation, M.S., J.M. and A.B.; writingoriginal draft preparation, M.S. and J.M.; writing-review and editing, A.B. and T.S.; supervision, J.M. and A.B.; project administration, M.S. and J.M. All authors have read and agreed to the published version of the manuscript.

Funding: This research received no external funding.

Institutional Review Board Statement: Not applicable.

Informed Consent Statement: Not applicable.

Data Availability Statement: Not applicable.

Conflicts of Interest: The authors declare no conflict of interest.

\section{References}

1. Kannan, R. Some results on fixed points. Bull. Calcutta. Math. Soc. 1968, 60, 71-76.

2. Chatterjea, S.K. Fixed point theorems. C. R. Acad. Bulgare Sci. 1972, 25, 727-730. [CrossRef]

3. Reich, S. Kannan's. Fixed point theorem. Boll. Dell'Unione Mat. Ital. 1971, 4, 459-465.

4. Cirić, L.B. A generalization of Bannach's contraction priciple. Proc. Am. Math. Soc. 1974, 45, 267-273. [CrossRef]

5. Reich, S. Some remarks concerning contraction mappings, canad. Math. Bull. 1971, 14, 121-124. [CrossRef]

6. Eldred, A.A.; Veeramani, P. Existance and converence of best proximity points. J. Math. Anal. Appl. 2006, 323, 1001-1006. [CrossRef]

7. Krapinar, E.; Erhan, I.M. Best on different Type Contractions. Appl. Math. Inf. Sci. 2011, 5, 558-569.

8. Petric, M.A. Best proximity point theorems for weak cyclic Kannan contractions. Filmat 2011, 25, 145-154. [CrossRef]

9. Wong, C. On Kannan maps. Proc. Am. Math. Soc. 1975, 47, 105-111. [CrossRef]

10. Todorcević, V. Harmonic Quasiconformal Mappings and Hyper-Bolic Type Metrics; Springer: Cham, Switzerland, 2019.

11. Radenović, S. Some remarks on mappings satisfying cyclical con- tractive conditions. Afr. Mat. 2016, 27, 291-295. [CrossRef] 
12. Zaslavski, A.J. Two fixed point results for a class of mappings of contractive type. J. Nonlinear Var. Anal. 2018, 2, 113-119.

13. Reich, S.; Zaslavski, A.J. Monotone contractive mappings. J. Nonlinear Var. Anal. 2017, 1, 391-401.

14. Kirk, W.A.; Srinivasan, P.S.; Veeramani, P. Fixed point fo mappings satisfyaing cyclical contractive conditions. Fixed Point Theory 2003, 4, 79-89.

15. Sabar, T.; Bassou, M.A. Best proximity point of tricyclic contraction. Adv. Fixed Point Ttheory 2017, 7, 512-523.

16. Sabiri, M.; Mouline, J.; Bassou, A.; Sabar, T. A New Best Approximation Result in (S) Convex Metric spaces. Int. J. Math. Math. Sci. 2020, 2020, 4367482. [CrossRef] 